\title{
Études/Inuit/Studies
}

\section{Le contrôle des chiens dans trois communautés du Nunavik au milieu du $20^{\mathrm{e}}$ siècle Control of dog populations in three Nunavik communities in the mid-20 ${ }^{\text {th }}$ century}

\section{Francis Lévesque}

Volume 34, numéro 2, 2010

URI : https://id.erudit.org/iderudit/1004074ar

DOI : https://doi.org/10.7202/1004074ar

Aller au sommaire du numéro

Éditeur(s)

Association Inuksiutiit Katimajiit Inc.

Centre interuniversitaire d'études et de recherches autochtones (CIÉRA)

ISSN

0701-1008 (imprimé)

1708-5268 (numérique)

Découvrir la revue

Citer cet article

Lévesque, F. (2010). Le contrôle des chiens dans trois communautés du Nunavik au milieu du $20^{\mathrm{e}}$ siècle. Études/Inuit/Studies, 34(2), 149-166.

https://doi.org/10.7202/1004074ar
Résumé de l'article

Le contrôle des chiens des Inuit par les autorités fédérales et provinciales au Nunavik fait l'objet d'un vif débat depuis plus d'une décennie. Depuis 1999, la Société Makivik accuse le gouvernement canadien et le gouvernement québécois d'avoir orchestré l'abattage des chiens du Nunavik dans les années 1950 et 1960 afin de favoriser l'assimilation des Inuit à la société canadienne. Pour leur part, les gouvernements d'Ottawa et de Québec ainsi que leurs corps policiers respectifs - Gendarmerie royale du Canada (GRC) et Sûreté du Québec (SQ) - nient catégoriquement avoir appliqué une politique d'élimination des chiens qui aurait eu pour objectif d'imposer un mode de vie nouveau aux Inuit. Cet article traite du contrôle des chiens au Nunavik durant cette période à partir d'événements survenus dans les communautés de Kuujjuaraapik, Puvirnituq et Kangirsujuaq.
Tous droits réservés @ La revue Études/Inuit/Studies,
Ce document est protégé par la loi sur le droit d'auteur. L'utilisation des services d’Érudit (y compris la reproduction) est assujettie à sa politique d'utilisation que vous pouvez consulter en ligne. 


\title{
Le contrôle des chiens dans trois communautés du Nunavik au milieu du 20e siècle
}

Francis Lévesque*

\begin{abstract}
Control of dog populations in three Nunavik communities in the mid-20th century
For over a decade, there has been emotionally charged debate over past efforts by the provincial and federal authorities in Nunavik to control the Inuit dog population. On the one hand, Makivik Corporation has claimed since 1999 that the federal and provincial governments had established a dog slaughter policy in Nunavik in the 1950s and 1960s as a way to facilitate assimilation of Inuit into Canadian society. On the other hand, both governments, the Sûreté du Québec (Quebec Provincial Police), and the Royal Canadian Mounted Police categorically deny having carried out such a policy with a view to imposing a new lifestyle on the Inuit. This article attempts to shed light on the fate of Inuit dogs in Nunavik during this period by looking at events in three settlements: Kuujjuaraapik, Puvirnituq, and Kangirsujuaq.
\end{abstract}

Résumé: Le contrôle des chiens dans trois communautés du Nunavik au milieu du 20e siècle

Le contrôle des chiens des Inuit par les autorités fédérales et provinciales au Nunavik fait l'objet d'un vif débat depuis plus d'une décennie. Depuis 1999, la Société Makivik accuse le gouvernement canadien et le gouvernement québécois d'avoir orchestré l'abattage des chiens du Nunavik dans les années 1950 et 1960 afin de favoriser l'assimilation des Inuit à la société canadienne. Pour leur part, les gouvernements d'Ottawa et de Québec ainsi que leurs corps policiers respectifs - Gendarmerie royale du Canada (GRC) et Sûreté du Québec (SQ) - nient catégoriquement avoir appliqué une politique d'élimination des chiens qui aurait eu pour objectif d'imposer un mode de vie nouveau aux Inuit. Cet article traite du contrôle des chiens au Nunavik durant cette période à partir d'événements survenus dans les communautés de Kuujjuaraapik, Puvirnituq et Kangirsujuaq.

* CIÉRA, Université Laval, Pavillon Charles-De Koninck, local 0450, 1030 avenue des SciencesHumaines, Québec, Québec G1V 0A6, Canada. francislevesque77@gmail.com

ÉTUDES/INUIT/STUDIES, 2010, 34(2): 149-166 


\section{Introduction}

Le contrôle des chiens des Inuit par les autorités fédérales et provinciales au Nunavik dans les années 1950 et 1960 fait l'objet d'un vif débat depuis plus d'une décennie. Dès 2000, la Société Makivik affirmait que «Durant la période allant de 1950 à 1975, le Canada et le Québec ont mis sur pied, par l'entremise de leurs représentants et de leurs forces policières, un programme d'abattage systématique des chiens des Inuit partout au Nunavik» (lettre de Pita Aatami, président de la Société Makivik à Guy Chevrette, ministre délégué aux Affaires autochtones, 28 mars 2000, in Société Makivik 2005a: Annexe 1, ma traduction). Cinq ans plus tard, la Société Makivik (2005b) ajoutait que «La perte d'attelages complets de chiens a eu une incidence importante sur les propriétaires: sans leur attelage, ils ne pouvaient plus partir à la chasse quand bon leur semblait. Leur liberté a été compromise. Une tradition a été sacrifiée. C'est, en quelque sorte, une forme de génocide qui a été perpétrée (sic) ici même au Canada». La Société Makivik a pressé les gouvernements du Canada et du Québec, dès 2000, de mettre sur pied une enquête publique afin de faire la lumière sur ces événements (Société Makivik 2005a: 1). Après plusieurs années d’attente déçue, la Société a finalement décidé de mettre sur pied sa propre commission d'enquête présidée par le juge Jean-Jacques Croteau, retraité de la Cour supérieure de justice du Québec. Il a rendu public son rapport final le 3 mars 2010 (Croteau 2010). Le rapport conclut que les gouvernements du Québec et du Canada ont manqué à leurs obligations fiduciaires envers les Inuit, entre autres parce que la Loi sur les abus préjudiciables à l'agriculture, utilisée par les autorités pour légitimer l'élimination de chiens errants, n’aurait pas dû être appliquée dans l'Arctique.

Pour leur part, les gouvernements d'Ottawa et de Québec et leurs corps policiers respectifs - la Gendarmerie royale du Canada (GRC) et la Sûreté du Québec (SQ) nient catégoriquement avoir appliqué une politique d'élimination des chiens qui aurait eu pour objectif d'imposer un mode de vie nouveau aux Inuit. Bien qu'ils concèdent que des chiens aient effectivement été abattus, ils affirment que seuls ceux qui représentaient un danger ou encore qui étaient malades l'auraient été. Le gouvernement canadien affirme même que, non seulement n'a-t-il jamais eu l'intention de tuer tous les chiens des Inuit, mais, qu'au contraire, il a fait tout son possible pour aider au maintien des populations canines en procédant à des campagnes de vaccination, en donnant de la nourriture à leur intention et en remplaçant les chiens décimés par de nouveaux (GRC 2006a, 2006b). ${ }^{1}$

Cet article explorera la question du contrôle des chiens au Nunavik dans les années 1950 et 1960. Il débutera par une mise en contexte de ce qui prévalait au Nunavik au milieu du 20e siècle. Il se poursuivra avec des récits au sujet du contrôle des chiens dans les communautés de Kuujjuaraapik, Puvirnituq et Kangirsujuaq. Il se terminera sur une discussion qui proposera quelques pistes de réflexion sur le sujet.

1 Voir aussi les lettres envoyées à Pita Aatami, président de la Société Makivik, par Guy Chevrette, ministre délégué aux Affaires autochtones du Québec le 21 juin 2000 et par Lawrence MacAuley, Solliciteur général du Canada 1er décembre 2000. Les deux lettres se trouvent dans Société Makivik (2005a) aux annexes 3 et 4 respectivement.

150/F. LÉVESQUE 


\section{La sédentarisation des Inuit et le «problème des chiens»}

Le contrôle des chiens survient à une époque où les conditions sociales, économiques, légales, politiques et même écologiques du Nunavik bouleversent la vie des Inuit. D'abord, c'est à ce moment que le gouvernement canadien renverse totalement ses politiques à leur égard. Jusqu'au milieu des années 1950, Ottawa décourageait les tentatives de sédentarisation des Inuit et insistait pour qu'ils poursuivent un mode de vie nomade axé sur la chasse, la pêche et la traite des fourrures (Damas 2002; Diubaldo 1985; Duhaime 1983). Après la Seconde Guerre mondiale, la valeur de la fourrure du renard arctique (Alopex lagopus) chute de $90 \%$, entraînant une baisse substantielle des revenus des Inuit. Au même moment, le caribou, dont ils dépendent pour se vêtir et se nourrir, disparaît subitement dans la plupart des régions de l'Arctique de l'Est canadien (Audet 1979). Les Inuit font face à une crise. Pour y faire face, le gouvernement canadien modifie ses politiques du tout au tout et décide de commencer à administrer l'Arctique et ses habitants. De 3,5 millions de dollars en 1953-1954, la part du budget du ministère du Nord canadien et des ressources nationales consacrées à l'administration des Inuit passe à 12 millions en 1956-1957 puis à 40 millions en 1959-1960².

L'objectif de ces mesures n'est pas uniquement d'aider les Inuit à surmonter la crise. Elles s'inscrivent à l'intérieur d'un projet colonial beaucoup plus vaste dont l'objectif avoué est d'intégrer les Inuit à la société canadienne en leur donnant les mêmes avantages qu'aux autres citoyens du pays (Lesage 1955; Robertson 1960, 1961). C'est ainsi qu'Ottawa financera la construction de maisons, encouragera les familles à envoyer leurs enfants à l'école (en les menaçant, entre autres, de leur retirer l'allocation familiale si elles ne le font pas) et mettra sur pied des infrastructures sanitaires. Si le gouvernement canadien n'impose pas la sédentarisation, ces mesures encouragent néanmoins les Inuit du Nunavik à venir s'établir dans les nouvelles communautés (Duhaime 1983).

Au tournant des années 1960, un autre revirement survient: le gouvernement québécois revendique le droit d'administrer lui-même le Nunavik et ses habitants. En juillet 1960, le parti libéral québécois dirigé par Jean Lesage—qui était quelques années plus tôt ministre du Nord canadien et avait présidé au renversement de politique du gouvernement fédéral à l'égard des Inuit—prend le pouvoir à Québec. Pour Lesage, il importe que le gouvernement québécois assure sa place dans l'Arctique ${ }^{3}$. Dès 1961, la SQ remplace la GRC dans les localités de Kuujjuaq et Kuujjuaraapik. En 1963, le Québec crée la Direction générale du Nouveau-Québec qui est placée sous la juridiction du ministère des Affaires naturelles dirigé par René Lévesque. En 1964, le Québec parvient à un accord avec Ottawa selon lequel il étendra tous ses programmes

2 ATNO, ASF, BF 17-3: Memorandum for all Staff: The Northern Administration Branch: Its New Organization, B.G. Sivertz, 5 janvier 1959. BF 18-12: A History of the Department of Northern Affairs and National Resources, R.T. Flanagan, vers 1966. BF 25-2: The Administration of Arctic Canada, R.A.J. Phillips, vers 1958.

3 BAC RG85, volume 1451, dossier 1006-8, pt. 2, 27 décembre 1962. 
aux localités du Nunavik. L'objectif du gouvernement québécois ne diffère en rien de celui du gouvernement fédéral: il tient à favoriser l'intégration des Inuit à la société québécoise. Ce faisant, il met sur pied des mesures qui encouragent leur sédentarisation et favorisent leur assimilation.

La sédentarisation des Inuit a plusieurs conséquences: perte de repères culturels, diminution du nombre d'animaux disponibles pour la chasse près des communautés, etc. Une de ces conséquences est la concentration de nombreux chiens dans les communautés. En effet, ce n'est pas parce qu'ils se sédentarisent que les Inuit renoncent à se déplacer et à chasser ${ }^{4}$. Il n'est pas rare de retrouver dans les communautés un nombre très important de chiens qui, de plus, sont rarement attachés puisque les Inuit considèrent que cela favorise leur socialisation. Cette dernière est importante puisque le chien inuit (canis familiaris borealis) peut présenter un danger pour les êtres humains, surtout ceux qu'il ne connaît pas (Freuchen 1935: 158; Qumaq 1992: 348). En outre, les Inuit tiennent à ce que leurs chiens soient libres de trouver leur pitance. Traditionnellement, les chiens suppléaient à la nourriture fournie par leur maître en mangeant les rebuts ou en chassant eux-mêmes de petits animaux. Lorsqu'ils se sédentarisèrent, les Inuit conservèrent cette habitude et laissèrent leurs chiens errer. C'était d'autant plus important que les Inuit qui occupaient des emplois n'avaient pas le temps de chasser assez pour nourrir tout leur attelage et qu'ils n'avaient pas les moyens de le nourrir avec de la nourriture achetée. La Compagnie de la Baie d'Hudson (CBH) vendait bien des sacs de nourriture sèche au coût de $1,05 \$$ chacun, mais il fallait trois sacs pour nourrir un attelage de 10 chiens quotidiennement (GRC 2006a: 214), pour un coût total de 22,05\$ par semaine (1146,60\$ annuellement). Une somme astronomique pour les Inuit. Enfin, il est important de noter qu'il n'était pas rare que le magasin de la CBH n'ait tout simplement pas en stock de chaînes et de colliers adéquats ${ }^{5}$ et que les Inuit devaient alors attacher leurs chiens avec des cordes ou de mauvaises chaînes que les chiens brisaient facilement.

Bref, au moment de la sédentarisation, des populations importantes de chiens errent dans chacune des communautés. Ces larges populations sont responsables de pillages et d'attaques contre des êtres humains. Entre 1957 et 1964, quatre enfants sont tués et plusieurs adultes attaqués au Nunavik seulement (Lévesque 2008: 346). Le nombre élevé de chiens errants favorise aussi la transmission de maladies canines telles que la rage, la maladie de Carré et l'hépatite canine infectieuse. Des épidémies éclatent d'ailleurs au début des années 1960 dans toutes les communautés de l'Arctique de l'Est et du Nunavik où elles tuent des centaines de chiens (Lévesque 2008: 256-267). Bien qu'elles ne soient pas inédites, ces épidémies sont les plus importantes de l'histoire canadienne et sont vraisemblablement liées à la sédentarisation des Inuit et à la concentration des chiens (Choquette et Moynihan 1964: 264).

4 La motoneige fera son apparition dans l'Arctique vers 1962 ou 1963, soit quelques années après les événements relatés ici. Elle coûtait alors $900 \$$, une somme importante pour les Inuit dont les sources de revenus se limitaient à des emplois journaliers, à la traite et à l'allocation familiale. En 1964, les revenus liés à l'allocation familiale pour les familles inuit du Nunavik se limitaient en moyenne à 146\$ annuellement (Jenness 1964: 150-151).

5 BAC RG85, volume 1959, dossier A-1006-8-1, partie 1, 19 octobre 1960.

\section{2/F. LÉVESQUE}


Pour le gouvernement canadien, il n'existe qu'une seule manière vraiment efficace de faire face à ce qu'il nomme le "problème des chiens» (dog problem) au Nunavik: exiger, comme dans les Territoires du Nord-Ouest (T.N.-O.), que les propriétaires attachent leurs chiens et permettre à la GRC de tuer ceux qui errent. ${ }^{6}$ Par contre, le Québec ne possède pas de loi qui s'adresse spécifiquement aux chiens errants et le gouvernement fédéral n'a pas l'autorité de légiférer à cet effet. Il doit donc se rabattre sur la législation existante. Malgré l'absence de loi spécifiquement destinée aux chiens errants, la province possède certaines dispositions légales dans la Loi sur les abus préjudiciables à l'agriculture (L.R.Q., chapitre A-2). L’article 11 spécifie: «Il est interdit au propriétaire, possesseur ou gardien d'un chien de le laisser errer dans un territoire non organisé, entre le premier mai et le quinze décembre.» L'article 12 précise que «Toute personne peut abattre un chien trouvé errant en contravention à l'article 11.»

Cette loi limite les possibilités d’intervention durant toute la période hivernale. Cela contrarie le gouvernement fédéral qui juge que cette restriction met la vie des habitants du Nunavik en danger. Malgré ses demandes répétées pour que le gouvernement québécois modifie la loi $^{7}$, Ottawa est incapable de le convaincre. C’est uniquement lorsqu'il commence à administrer le Nunavik que le gouvernement québécois apporte des modifications qui lui permettront de tuer les chiens errants à longueur d'année. Le 23 février 1965, l’Arrêté 332 est voté. Il stipule que:

Dans les localités de Poste-de-la-Baleine, Inoudjouac (Port-Harrison), Povungnituk, Ivuyivik, Saglouc, Baie-Wakeham, Koartak, Baie Payne, Fort-Chimo, Port-NouveauQuébec (George River), pour une période de dix (10) ans, il est interdit au propriétaire, possesseur ou gardien d'un chien de le laisser errer sous peine d'une amende de cinq dollars au moins et de vingt-cinq dollars au plus. Toute personne peut tuer, sans encourir aucune responsabilité, tout tel chien trouvé errant ainsi illégalement dans ces localités (Gazette officielle du Québec, 13 mars 1965, vol. 11).

En 1967, la politique québécoise à l'égard du contrôle des chiens inuit change de nouveau. Selon Alexander Stevenson, l'administrateur de l'Arctique de 1963 à 1970, la police provinciale de Kuujjuaq, «en accord avec la législation provinciale concernant le contrôle des chiens, avait procédé à la destruction de chiens errants jusqu'à récemment.» Cependant, «cette pratique a cessé suite à des ordres reçus de Québec. La raison donnée pour justifier cette décision était qu’il fallait cesser de contrarier les Inuit» ${ }^{8}$.

6 Les T.N.-O. possèdent un Arrêté sur les chiens (Dog Ordinance) adopté en 1929 qui permet à des officiers nommés par le Commissaire du territoire de capturer et tuer les chiens errants et de poursuivre les propriétaires reconnus coupables d'avoir laissé errer leurs chiens (Lévesque à paraître).

7 BAC RG22, volume 334, dossier 40-8-1, partie 10, 17 novembre 1958. ATNO, ASF, BF 35-19, Communities - Arctic Quebec 1955-1972, «Memorandum for Mr. Stevenson. Control of Dogs in Quebec Province», W.G. Kerr, 3 juin 1960.

8 BAC, RG85, versement 1997-98/076, boîte 159, dossier: 1006-8-1, Control of Dogs, 17 octobre 1967, ma traduction. 
C'est donc dans ce contexte que se déroule le contrôle des chiens des Inuit du Nunavik. Sur le terrain cependant, le contrôle des chiens se traduit de manière différente d'une communauté à l'autre. Les trois récits présentés ici permettront de rendre compte de cette diversité.

\section{Le contrôle des chiens dans trois communautés du Nunavik}

Les trois récits présentés ici s’appuient à la fois sur des données qui proviennent de recherches en archives ainsi que sur des témoignages d'aînés inuit recueillis par la Société Makivik entre 1999 et 2000. La somme de données archivistiques ou orales disponibles pour chacune des communautés diffère grandement. Les deux premiers récits s'attardent aux communautés de Kuujjuaraapik et Puvirnituq. Ils s'appuient sur des données archivistiques puisque la Société Makivik ne m’a transmis que deux entrevues au sujet de Puvirnituq et une seule au sujet de Kuujjuaraapik. Le récit de Kangirsujuaq s'appuie presque uniquement sur des témoignages inuit transmis par la Société Makivik. Je possède très peu de données archivistiques concernant cette communauté.

\section{Kuujjuaraapik}

Kuujjuaraapik est située à l'embouchure de la Grande rivière de la Baleine. À partir de 1955, les États-Unis construisent des infrastructures militaires là où la Compagnie de la Baie d'Hudson avait établi un poste presque 100 ans auparavant. En 1958, le gouvernement fédéral envoie ses premiers fonctionnaires et bâtit la première école. En 1961, la SQ remplace la GRC. En 1965, le gouvernement québécois reprend les locaux laissés vacants par l'armée canadienne. En 1967, la population de la communauté est estimée à environ 885 personnes (500 Inuit, 260 Cris et 125 nonAutochtones). ${ }^{9}$

C'est en 1957 que surviennent les premiers incidents liés aux chiens dans la communauté. En avril de cette année-là, Donald B. Marsh, alors évêque anglican du diocèse de l'Arctique, s'adresse à Gordon Robertson, qui occupe la double fonction de Commissaire des Territoires du Nord-Ouest et de sous-ministre du ministère du Nord et des Ressources nationales. Dans sa lettre, l'évêque demande à Robertson d'imposer aux Inuit qu'ils attachent leurs chiens, car certains ont blessé la fille du missionnaire anglican de Kuujjuaraapik ainsi qu'une jeune fille $\mathrm{crie}^{10}$. L'intervention de l'évêque incite Robertson à questionner R.L. Kennedy, le NSO (Northern Service Officer) ${ }^{11}$ de

9 ATNO, ASF, BF 35-16, Communities - Great-Whale-River, Quebec 1955-1958, «Great-Whale-River (Poste-de-la-baleine) P.Q.», décembre 1967.

10 BAC RG22, volume 1332, dossier 40-2-170, lettre de M. Marsh à M. Robertson, 12 avril 1957.

11 Les NSO sont les fonctionnaires fédéraux chargés d'administrer les programmes du gouvernement canadien dans les communautés nordiques.

\section{4/F. LÉVESQUE}


Kuujjuaraapik, au sujet des chiens dans la communauté 12 . Kennedy répond avoir déjà discuté de la question avec le sergent-détective Paul Émile Tourville de la police provinciale l'été précédent et qu'ils avaient convenu d'appliquer la Loi québécoise sur les abus préjudiciables à l'agriculture ${ }^{13}$. Kennedy ajoute aussi dans cette missive que la fille du missionnaire anglican avait été attaquée par un chien déjà attaché.

En septembre 1957, plusieurs chiens errants détruisent un dépôt de nourriture de la station de radar. Un policier de la GRC se charge de tous les tuer. Le 8 septembre, Kennedy convoque tous les propriétaires de chiens. Les Inuit dont les chiens ont détruit le dépôt en profitent pour se plaindre du traitement réservé à leurs bêtes. En vain. Kennedy ne fait que leur rappeler les provisions de la loi québécoise qui les oblige à garder leurs bêtes attachées durant cette période de l'année. Suite à la réunion, le policier de la GRC rédige un rapport dans lequel il mentionne que tous les Inuit qui se sont plaints d'avoir perdu un chien ont admis qu'ils avaient été avertis de les attacher sous peine de les voir mourir ${ }^{14}$. Il rejette donc la responsabilité de la mort des chiens sur les épaules de leurs propriétaires.

En septembre 1958, une jeune Inuk est attaquée dans un camp situé près du golfe de Richmond, à trois kilomètres au nord de Kuujjuaraapik. Malgré qu'elle ait été immédiatement transférée dans un hôpital de Val-d'Or, elle meurt des suites de ses blessures. Cet accident, jumelé à celui survenu l'année précédente, inquiète R.A.J. Phillips, le chef de la Division de l'Arctique. Dans un mémorandum envoyé au nouveau NSO de Kuujjuaraapik, J.G. Walton, Phillips exige que des mesures soient prises pour que de tels accidents ne se reproduisent plus ${ }^{15}$. Walton lui répond qu'il sera difficile d'imposer de telles mesures aux Inuit, puisque ces derniers acceptent les risques liés au fait de ne pas attacher les chiens ${ }^{16}$.

Néanmoins, Walton convoque une réunion où il demande aux Inuit d'attacher leurs chiens en tout temps pour prévenir les accidents. Les Inuit sont réticents. Pour eux, cela signifie qu'ils doivent passer plus de temps à la chasse, ce qui implique s'éloigner de leur famille et potentiellement perdre des revenus. Le commandant de la base militaire suggère alors de donner aux chiens les restes de table de la base. Si les non-Inuit de la communauté sont ravis de cette proposition, les Inuit se montrent, une fois encore, réticents. En effet, plusieurs pensent que cette nourriture est empoisonnée et refusent de

12 BAC RG85, versement 1999-98/076, volume 159, dossier 1006-8-1, Memorandum for Mr. Kennedy, 17 mai 1957.

13 BAC RG85, versement 1999-98/076, volume 159, dossier 1006-8-1, Control of Dogs Running Loose in Northern Quebec, 8 octobre 1958.

14 BAC RG85, versement 1999-98/076, volume 159, dossier 1006-8-1, Control of Dogs Running Loose in Northern Quebec, 8 octobre 1958.

15 BAC RG85, versement 1999-98/076, volume 159, dossier 1006-8-1, Memorandum for Mr. Walton, 3 octobre 1958. Voir aussi Hamilton 2002.

16 BAC RG85, versement 1999-98/076, volume 159, dossier 1006-8-1, Memorandum for the Chief, Arctic Division, 3 octobre et 4 octobre 1958.

LE CONTRÔLE DES CHIENS DANS TROIS.../155 
la donner à leurs chiens ${ }^{17}$. Un Inuk affirme même que ses chiens sont morts d'en avoir mangée (M-015) ${ }^{18}$.

Au milieu du mois de mai de 1959, le sergent-détective Tourville revient dans la communauté et impose que tous les chiens soient attachés dans les 24 heures. Il prévient que, suite à cet ultimatum, les chiens errants seront systématiquement tués. Dans un mémorandum envoyé à l'administrateur de l'Arctique, Walton souligne qu'il

est dommage que des mesures si draconiennes soient prises. Cependant, la non-coopération de certains Eskimos ne nous laisse pas le choix. À plusieurs occasions, le contrôle des chiens a fait l'objet de discussions avec les Eskimos de la communauté: certains ont coopéré, mais d'autres pas. [...] Pour cette raison, la police n'avait d'autre alternative que de détruire les chiens. ${ }^{19}$

Malgré cette menace, une majorité d’Inuit continue de ne pas attacher ses chiens. Certains se justifient en affirmant qu'il s'agit là du seul moyen pour que leurs chiens se nourrissent, d'autres affirment que les chiens ne sont dangereux que lorsqu'ils sont attachés.

Le 22 juillet 1959, William Shakleton, le contrôleur de la station de radar, tue deux chiens errants ${ }^{20}$. Furieux, leurs propriétaires décident d'aller le confronter à son camp. Selon Walton, ce n'est pas le fait que Shakleton ait tué deux chiens qui rend les Inuit furieux, mais plutôt le fait que:

M. Shakleton a été négligent lorsqu'il s'est mis à tirer des coups de feu dans la communauté. Je pense que ce sont les coups de feu qui ont percé les tentes qui ont causé du ressentiment, pas le fait que certains chiens aient été tués. Ce n’est pas la première fois que des chiens sont tués à Poste-à-la-Baleine, mais c'est la première fois que les Eskimos semblent si inquiets ${ }^{21}$.

Le jour suivant, Shakleton envoie un télégramme à Maurice Duplessis, premier ministre du Québec, où il explique que les chiens errants représentent une menace grave pour les habitants de la communauté. Trois semaines plus tard, le sergent-

17 BAC RG85, versement 1999-98/076, volume 159, dossier 1006-8-1, Memorandum for the Chief, Arctic Division, 3 octobre 1958.

18 Pour des raisons de confidentialité liées à l'entente que j’ai signée avec la Société Makivik, je dois préserver l'anonymat des témoins inuit. J’utilise des numéros pour cette raison. Le «M» signifie que les entrevues ont été efectuées par les chercheurs de la Société Makivik.

19 BAC RG85, versement 1999-98/076, volume 159, dossier 1006-8-1, Memorandum to the administrator of the Arctic, 19 avril 1959, ma traduction.

20 La fille de Shakleton avait succombé à ses blessures après avoir été attaquée par des chiens à Puvirnituq deux ans auparavant (GRC 2006a: 356).

21 BAC RG85, versement 1999-98/076, volume 159, dossier 1006-8-1, Memorandum for the Administrator of the Arctic, 6 octobre 1959, ma traduction.

156/F. LÉVESQUE 
détective Edgar Anderson de la police provinciale vient enquêter sur cette affaire. Lors de son séjour, il tue six chiens errants ${ }^{22}$.

Non content d'avoir alerté les autorités provinciales, Shakleton médiatise l'affaire. À la fin d'août 1959, la Gazette de Montréal publie un entrefilet tiré d'une missive adressée par lui à sa rédaction (Anonyme 1959a). Le même mois, le Time Magazine publie un autre entrefilet qui affirme que «des huskies affamés, gardés en liberté par les Eskimos [...] terrorisaient le village. Quand Shakleton a pris son arme et a tué des chiens pour protéger sa famille, les Eskimos ont répliqué en tirant des coups de feu à sa cabane de bois» (Anonyme 1959b, ma traduction).

L'administration de l'Arctique s'inquiète du conflit émergent. Cette inquiétude est palpable dans un mémorandum envoyé à Walton par C.M. Bolger, alors administrateur de l'Arctique. Dans ce mémorandum, Bolger rappelle qu'il est très important de trouver une solution adéquate au problème des chiens, car ces derniers «représentent une partie importante de leur culture, autant comme moyen de transport que comme symbole de prestige et de succès» (ma traduction). Bolger poursuit en exposant la stratégie de l'administration canadienne face aux chiens errants:

La solution à long terme concernant le problème des chiens errants est de convaincre leurs propriétaires de les garder attachés. Le fait de tirer sur tous les chiens sans distinction réduit certes leur nombre, mais augmente l'hostilité des Eskimos à l'égard de la personne qui a tué le chien et du groupe auquel elle appartient. Après cela, il est plus difficile de convaincre les Eskimos de l'importance d'attacher leurs chiens ${ }^{23}$.

Deux ans plus tard, soit à la fin de l'été 1961, un nouveau problème fait son apparition: une cinquantaine de chiens meurent victimes de la rage et de la maladie de Carré (Choquette et Moyhinan 1964: 264). Pour enrayer la propagation de ces maladies, le ministère de l'Agriculture recommande de garder les chiens attachés, d'en vacciner le plus possible et de tuer ceux qui sont déjà malades. ${ }^{24}$ En décembre 1962, certains chiens contractent une maladie que l'on pense être la rage. Ces chiens sont immédiatement tués par les agents de la Sûreté du Québec et l'une des bêtes abattues est envoyée à l'Institut de recherche sur les maladies animales de Hull pour analyse. Dans le communiqué qui accompagne le cadavre de l'animal, David C. Neve, l'administrateur nordique responsable de la région de Kuujjuaraapik, affirme que «ces chiens sont extrêmement importants pour les Eskimos et je suis prêt à tout faire pour empêcher la propagation de maladies» ${ }^{25}$.

22 BAC RG85, versement 1999-98/076, volume 159, dossier 1006-8-1, Memorandum to the Arctic Administration, 4 septembre 1959.

23 BAC RG85, versement 1999-98/076, volume 159, dossier 1006-8-1, Memorandum to the Arctic Administration, 4 septembre 1959, ma traduction.

24 BAC RG85, volume 1959, Dossier A-1006-8-1, partie 1, Memorandum for the Area Administrator, 27 mars 1961.

25 BAC RG85, volume 1959, Dossier A-1006-8-1, partie 1, Lettre de David C. Neve à l'Officier en charge de l'Institut de recherche sur les maladies animales de Hull, 11 décembre 1962, ma traduction. 
Le problème est si aigu que l'administration canadienne envoie un vétérinaire dans la communauté pour évaluer la situation. Cette visite lui permet de constater que les chiens sont victimes de l'hépatite canine infectieuse. Rien qu'en décembre, la maladie tue une quarantaine de chiens. Il profite aussi de sa visite pour vacciner 250 chiens (GRC 2006a: 240). Pour contrer l'épidémie, l'administration nordique envoie 2350 doses de vaccins au Nunavik à l'hiver 1963 (GRC 2006a: 245) en plus de 550 autres l'année suivante ${ }^{26}$. En 1966, la rage refait surface. L'administration canadienne décide d'envoyer plus de 300 doses de vaccins dans la communauté ${ }^{27}$. Néanmoins, elle ne parvient pas à sauver tous les chiens. Au début des années 1970, il ne restait plus de chiens inuit dans la communauté et les Inuit utilisaient désormais tous la motoneige (M-015).

\section{Puvirnituq}

C'est à partir de 1951, alors que la Compagnie de la Baie d'Hudson y ouvre un poste, que des Inuit commencent à se regrouper à Puvirnituq (GRC 2006a: 345; Audet 1974: 8; 85-86). Le nombre d'Inuit augmentera ensuite assez rapidement, entre autres parce que plusieurs décident de s'y établir en attendant leurs parents envoyés dans le Sud pour subir des traitements contre la tuberculose (Audet 1974: 8). En 1956, une mission catholique s’y établit. Dans les années 1960, la communauté compte près de 500 habitants, Inuit pour la plupart ${ }^{28}$.

Au début des années 1960, il y a à Puvirnituq plus de chiens que d'êtres humains (Mallon s.d.). Malgré cela, les chiens ne semblent pas représenter un problème important. Mis à part l'accident non documenté de la fille de William Shakleton en 1957 (voir note 20), aucun autre incident n'est rapporté ni par les documents d'archives, ni par les aînés. C'est en 1963, avec l'apparition de maladies canines, que la situation se complique. Au cours de l'hiver de cette année-là, on découvre un premier chien malade. Ce chien, écrit le NSO de Puvirnituq, J.D. Furneaux, était en bonne santé jusqu'au 31 décembre 1962. Cependant, sa condition se dégrade rapidement. Lorsqu'il commence à attaquer les autres chiens de son attelage, il est aussitôt abattu. Furneaux précise que d'autres chiens présentant des symptômes similaires ont dû être tués. Il suppose que les chiens sont morts de la rage, mais comme il n'en est pas certain, il envoie la tête d'un des animaux à Ottawa pour analyse ${ }^{29}$. Bien que les résultats de ces tests demeurent inconnus, il est probable qu'il se soit agi de la maladie de Carré ou encore de l'hépatite canine infectieuse, qui affectait les chiens d'autres communautés du Nunavik et de l'île de Baffin au même moment.

26 BAC RG85, volume 1959, dossier A-1006-8-1, partie 1, Memorandum for the administrator of the Arctic, 29 avril 1963.

27 BAC RG85, versement 1997-98/076, boîte 159, dossier 1006-8-1, Rabies Outbreak - Great Whale River, 28 janvier 1966.

28 BAC RG85, volume 1959, Dossier A-1006-8-1, partie 1, Memorandum for the Quebec regional Administration. Dogs for Breeding, 10 juin 1963.

29 BAC RG85, volume 1959, Dossier A-1006-8-1, partie 1, Dog's Head for Examination, 2 janvier 1963.

\section{8/F. LÉVESQUE}


D’après l'épouse de Frank Vallee ${ }^{30}$, deux personnes sont attaquées par des chiens au moment même où l'épidémie se déclare dans la communauté. Dès cet instant, les chiens qui montrent des signes de maladie sont systématiquement tués. Ainsi, au mois de février 1963, elle pense que près de 70 chiens malades l'ont déjà été. C'est le conseil du village qui en a décidé ainsi. Lors de réunions tenues en janvier et février, les membres du conseil (autant les Inuit que les non-Inuit) décident que tous les chiens malades et errants doivent être tués afin d'éviter les risques de propagation des maladies. Pour éviter que tous les habitants ne se lancent à la poursuite des chiens, le conseil décide de nommer deux personnes dont le mandat sera de les tuer. Mallon raconte que les Inuit réfléchissent longuement à l'identité des deux candidats qui agiront à titre de patrouilleurs. Ils suggèrent entre autres Frank Vallee, «ce Qallunaaq qui ne fait rien d'autre que de se promener et de poser des questions» (Mallon s.d., ma traduction). L'épouse de Vallee raconte comment se déroulaient les patrouilles:

Chaque matin vers 9h30, Frank et Johnny Angitugulu POV ${ }^{31}$, l'Eskimo membre de la patrouille, prennent leur fusil et patrouillent la communauté. [...] Depuis qu'elle a été lancée il y a sept jours, la patrouille a tué quatre chiens. Deux avaient la rage et furent faciles à tuer puisqu'ils étaient attachés. Les deux autres cependant avaient brisé leurs liens et coururent dans toutes les directions entre les cabanes et les iglous, où il était trop dangereux de les tirer. Ils furent finalement abattus à l'orée du village ${ }^{32}$.

Selon les estimations de Furneaux, l'épidémie de 1963 tue entre 60 et 100 chiens. ${ }^{33}$ Pour pallier cette perte, plusieurs Inuit lui demandent s'il est possible d'importer des chiens d'autres régions. Furneaux fait donc la demande à l'administration québécoise qui refuse sa requête sous prétexte que les Inuit sont responsables de la mort de leurs propres chiens. Ce refus suscite une vive réaction de Furneaux qui leur répond que:

Comme pour les maladies qui affectent les humains, il ne faut pas s'imaginer que les maladies canines sont causées par de la négligence. Les Eskimos de la communauté ne négligent pas leurs chiens. Au contraire, presque tous donnent une attention toute particulière à leurs attelages. [...]

La raison pour laquelle j'ai demandé d'avoir de nouveaux chiens est que l'épidémie de rage a eu pour effet de causer un manque de chiens pour les chasseurs de Puvungnituk. Je voulais être certain que les chiens qui allaient les remplacer étaient de bonne souche ${ }^{34}$.

30 L'anthropologue Frank Vallee se trouvait dans la communauté avec son épouse au moment des événements rapportés ici. Cette dernière, dont le nom m’est inconnu, les a racontés dans une lettre envoyée à sa famille qui se trouve à BAC RG85, volume 1959, dossier A-1006-8-1, partie 1, 15 février 1963.

31 Juani POV Angutiguluk Novaligna était un Inuk influent de la région de Puvirnituq. Il est décédé en 1978. Comme d'autres Inuit de la même communauté, notamment l'artiste Abraham POV, il écrivait son patronyme en lettres majuscules. Ce dernier est une contraction du nom de la communauté et se prononce «piouvi» («P.O.V.» épelé en anglais).

32 BAC RG85, volume 1959, dossier A-1006-8-1, partie 1, 15 février 1963, ma traduction.

33 BAC RG85, volume 1959, Dossier A-1006-8-1, partie 1, Memorandum for the Quebec Regional Administrator. Dogs for Breeding, 14 mai 1963.

34 BAC RG85, volume 1959, Dossier A-1006-8-1, partie 1, Memorandum for the Quebec Regional Administrator. Dogs for Breeding, 10 juin 1963, ma traduction. 
Suite à cet épisode, les Inuit adoptent la motoneige. Dès 1965, la majorité des Inuit de cette communauté l'utilise (Audet 1974: 37). Malgré cela, des chiens continuent à peupler la communauté, car certains Inuit racontent que la Sûreté du Québec en tua un nombre important en 1968 (Qumaq 1992: 348).

\section{Kangirsujuaq}

Dans les années 1960, la communauté de Kangirsujuaq ne compte que 187 habitants, 176 Inuit pour 11 non-Inuit (Saladin d'Anglure 1967). Aucun agent de police n’y est stationné. Jusqu'au milieu des années 1960, les chiens errants ne semblent pas y représenter un enjeu important: ni les aînés inuit ni les documents consultés dans les archives ne font état d'incidents liés aux chiens errants dans cette communauté.

En février 1965 ou 1966 cependant, un événement marquant survient à Kangirsujuaq. Plusieurs Inuit racontent que quelques mois après que deux femmes aient été attaquées par des chiens, un policier de la SQ et un Inuk d'Inujjuaq sont venus dans la communauté pour tuer les chiens. Dès leur arrivée, ils convoquent une réunion où ils demandent aux Inuit d'amener leurs chiens inutiles sur une petite île au large de la communauté dès le lendemain. Ils leur précisent alors que ces chiens seront tués. Surpris, les Inuit obtempèrent néanmoins. Le policier et son adjoint tuent un nombre important de chiens et demandent à plusieurs Inuit d'en tuer aussi en utilisant leurs propres munitions. Les cadavres des animaux sont ensuite arrosés d'essence, brûlés et abandonnés sur la banquise. Plusieurs Inuit témoignent de cet épisode. Selon un Inuk,

Cela s'est déroulé entre 1965 et 1967. C’était la Sûreté du Québec. J'étais l’interprète du policier à ce moment. Le policier nous a dit que les chiens seraient tués parce qu'il y avait un risque qu'ils aient la rage. Nous avons eu une rencontre à ce sujet, et les Inuit de la communauté n'ont pas réagi. Le policier travaillait avec un Inuk de Kuujjuaq. Nous devions tous amener nos chiens, même si nous ne le voulions pas. Ils ont entraîné les chiens sur une île où ils les ont tués. Les chiens de mon père ont été tués. Je me souviens encore de la pile de carcasses sur la glace (M-019, ma traduction).

Il ne semble pas que l'intention du policier fût de tuer tous les chiens. Cependant, il forçait les Inuit à tuer les chiens qu'ils n'utilisaient plus: «Ils m’ont dit que si je n'avais plus besoin de mes chiens et que si ma motoneige fonctionnait, ils voulaient tuer mes chiens. J'ai accepté, et ce, même si je souhaitais les garder au cas où ma motoneige briserait» (M-043, ma traduction). Lorsque le policier demande aux Inuit d'amener leurs chiens sur la plage, ces derniers obtempèrent: «Les chiens [...] étaient sous harnais et ils furent amenés par leur maître parce qu’on leur a ordonné de le faire. Les maîtres avaient accepté le fait que leurs chiens allaient mourir» (M-040, ma traduction). L'adjoint inuit du policier confirme lui-même la participation de la majorité des Inuit à cette opération: «Les gens nous amenaient leurs chiens quand nous étions sur la glace à Kangiqsujjuaq (sic). Les gens nous ont amené leurs chiens. Je suppose que c'est ce qu'ils voulaient puisqu'ils nous les amenaient» (M-097, ma traduction).

160/F. LÉVESQUE 
En plus de choisir les chiens et de les amener à la plage, plusieurs Inuit ont euxmêmes dû tuer leurs chiens avec leurs propres munitions. En effet, «Ces hommes étaient censés tuer les chiens, mais mon père dut tuer ceux de ses chiens qu'il appréciait le moins en utilisant son propre fusil et ses propres munitions. Ils [les Inuit] tuaient leurs propres chiens sous la supervision du policier» (M-034, ma traduction). Après l'abattage de ces chiens, il fallut disposer de leurs cadavres. Un Inuk raconte que:

Ils [le policier et son adjoint] ont formé une haute pile de cadavres sur laquelle ils ont versé de l'essence puis ils y ont mis le feu. La pile devait faire environ cinq ou six pieds. Tous ces chiens n'ont pas été tués en une seule journée, mais en deux ou trois jours. Les cadavres ont été brûlés et laissés sur la glace. Nous continuions à les voir, même s’ils étaient brûlés (M033, ma traduction).

Une Inuk raconte sa tristesse au moment où les chiens furent amenés sur la glace pour être tués d'abord et brûlés ensuite. Elle raconte aussi comment son propre mari avait, comme bien d'autres hommes d'ailleurs, amené ses chiens au policier:

Les chiens ont été attachés puis amenés à l'endroit où ils allaient être tués. Je ne pouvais regarder quand ils tuaient les chiens parce qu'ils me manquaient déjà. [...] Mon époux était un de ceux qui avaient amené ses chiens, comme plusieurs autres hommes. Même si nous ne sommes pas des enfants, nous avons pleuré, surtout que même les chiens de nos enfants sont morts (M-031, ma traduction).

Si le séjour du policier et de son adjoint mène à la mort de plusieurs chiens dans la communauté, tous ne sont pas victimes du même sort. Selon un Inuk, au moins la moitié des chiens furent épargnés (M-034). Plusieurs affirment avoir pu voyager et aller à la chasse en traîneaux par la suite. D'ailleurs, le policier et son adjoint ont été reconduits à l'avion qui venait les chercher en traîneau à chien. Si tous les chiens n'ont pas été tués, cet épisode fut néanmoins très douloureux et marqua l'imaginaire des habitants de la communauté. Suite à cet épisode, aucun événement relatif au contrôle des chiens ne semble être survenu dans la communauté.

\section{Discussion et conclusion}

Suite à ce qui a été mentionné jusqu'ici, il est possible d'en arriver à certains constats au sujet du contrôle des chiens au Nunavik dans les années 1950 et 1960. Les Inuit ont souffert de cette mesure. D’abord, parce qu'avec un nombre réduit de chiens, il leur était difficile de poursuivre leurs activités de chasse et de trappe, et de voyager sur le territoire. Ensuite, parce que les chiens éliminés étaient des membres à part entière de la société. Dans la culture inuit, les chiens sont les seuls animaux à posséder un atiq, ou âme-nom. L'atiq est une entité autonome et immortelle qui véhicule un ensemble de qualités, de capacités et de désirs (Saladin d'Anglure 1977: 50). C’est l'atiq qui confère à celui qui le porte un statut social défini au sein de la société. Parce qu'il porte un atiq, le chien fait donc partie intégrante de la société (Laugrand et Oosten 2002: 101). Quand des policiers ont tué leurs chiens, les Inuit ont donc perdu des membres de leur société. Enfin, le propriétaire et ses chiens formaient une entité 
symbolique. Pour les Inuit, tous les animaux possèdent un inua, c'est-à-dire un «propriétaire» ou «maître» (Laugrand et Oosten 2002: 101). Les animaux et leurs inua forment un tout symbolique, l'un ne pouvant exister sans l'autre. Le chien possède aussi un inua: son maître. Ils forment donc un tout symbolique. Lorsque des chiens ont été tués, les Inuit se sont sentis menacés, car le tout symbolique qu'ils formaient avec leurs chiens était brisé ${ }^{35}$.

Cela dit, ce n'est pas parce que les Inuit ont souffert et que les gouvernements fédéraux et provinciaux ont appliqué une loi qui allait mener à la mort de plusieurs chiens qu'il faut en déduire que ces mesures visaient à faire souffrir les Inuit. Il est important ici de ne pas confondre les intentions des autorités gouvernementales et leurs effets négatifs sur les Inuit. Les intentions des autorités gouvernementales — autant nationales, provinciales que locales — semblent avoir été de créer un milieu de vie sécuritaire pour les populations inuit et non inuit qui vivaient dans les communautés du Nunavik. En effet, dans les années 1950 et 1960, le gouvernement fédéral et le gouvernement provincial cherchent à instaurer partout au pays, autant dans le Sud que dans le Nord, des mesures liées à l'État providence qui cherchent à contrôler les risques potentiels qui peuvent mettre la sécurité des populations en danger. Ainsi, c'est à cette époque que les autorités gouvernementales reprennent l'éducation et la santé des mains de l'Église, qu'elles créent des programmes de logements sociaux, instaurent des normes sanitaires sévères, et mettent en place des mécanismes qui cherchent à gérer les risques inhérents au fait de vivre en société (voir Foucault 1991). Bref, c’est à ce moment que les divers paliers de gouvernements du Canada commencent à tisser un filet de sécurité autour des populations du pays. Et c'est ce filet que Jean Lesage (1955), Gordon Robertson $(1960,1961)$ et d'autres tentent d'étendre aux Inuit. Le contrôle des chiens s'inscrit dans cette logique: les chiens errants et malades représentent un risque pour ceux qui vivent dans les communautés de l'Arctique. Pour les protéger, il faut donc instaurer des mécanismes qui permettront de contrôler ce risque. Ces mécanismes comprennent l'adoption et l'application de mesures législatives qui obligent les Inuit à attacher leurs chiens et qui permettent aux autorités de tuer les chiens errants, la mise sur pied de campagnes de vaccination, ou encore le remplacement des chiens décédés par de nouveaux provenant d'autres régions de l'Arctique.

Les trois récits présentés plus haut permettent d'ailleurs de constater que le contrôle des chiens devient un enjeu uniquement lorsque la sécurité des populations locales est menacée. En effet, c'est lorsque les chiens sont responsables d'accidents ou encore lorsqu'ils sont menacés par des maladies qui représentent un risque pour la santé ou la sécurité des Inuit que la nécessité de les contrôler refait surface. À Kuujjuaraapik par exemple, c'est suite à un accident survenu en 1957 que le sujet émerge pour la première fois. Il n'en sera question la prochaine fois qu'à la mort de plusieurs chiens malades en 1961 et 1962. À Puvirnituq, c'est l'apparition de maladies qui encourage le conseil du village à former une patrouille dont l'objectif est d'éliminer les chiens

35 Pour une discussion plus détaillée au sujet de l'atiq, de l'inua et des chiens, voir Laugrand et Oosten (2002) ainsi que Lévesque (2008), chapitre 2 et 7, et Lévesque (à paraître).

\section{2/F. LÉVESQUE}


potentiellement nuisibles. À Kangirsujuaq, il semble que ce soit l'attaque de deux femmes par des chiens survenue quelques mois avant l'épisode relaté plus haut qui a encouragé les autorités provinciales à vouloir éliminer les chiens «inutiles» (Lévesque 2008: 328).

Le contrôle des chiens survient lorsque les autorités, qu'elles soient fédérales, provinciales ou même communautaires, jugent que la sécurité des populations locales est menacée par des chiens dangereux ou malades. De plus, il s'inscrit dans une logique qui dépasse largement les frontières de l'Arctique québécois. Il ne s'agit donc pas d'une tentative de détruire ou de faire souffrir les Inuit, même si ce fut là l'effet que cette mesure eut sur les Inuit.

\section{Remerciements}

La recherche qui a mené à la rédaction de cet article a été financée par l'Alliance de recherche universités-communautés (ARUC) «Mémoires et histoires au Nunavut», le Fonds québécois de la recherche en science sociale (FQRSC), le Programme de formation scientifique pour le Nord (PFSN), le Fonds Georges-Henri-Lévesque (FGHL) ainsi que le Vice-President's Office de l'Université de l'Alberta. Je remercie Pita Aatami, président de la Société Makivik, d'avoir bien voulu partager les entrevues réalisées par la Société avec moi. Finalement, je tiens à remercier les deux évaluateurs anonymes pour leurs précieux commentaires.

\section{Sources d'archives}

ATNO (ARCHIVES DES TERRITOIRES DU NORD-OUEST)

-Fonds Alexander Stevenson.

BAC (BIBLIOTHÈQUE ET ARCHIVES, CANADA)

-RG22, volume 334, dossier 40-8-1, partie 10.

-RG85, versement 1999-98/076, volume 159, dossier 1006-8-1.

-RG85, volume 1451, dossier 1006-8, partie 2.

-RG85, volume 1959, dossier A-1006-8-1, partie 1.

LÉGISLATIONS

-Loi sur les abus préjudiciables à l'agriculture, L.R.Q., chapitre A-2.

-Arrêté 332, Gazette officielle du Québec, 13 mars 1965, vol. 11. 


\section{Références}

AUDET, Michel

1974 Le réseau spatial des Qikirtajuarmiut et l'ouverture d'un nouveau village à Akulivik, Mémoire de maîtrise, département d'anthropologie, Université Laval, Québec.

AUDET, René

1979 Histoire du caribou du Québec-Labrador et évolution des populations, Recherches amérindiennes au Québec, 9(1-2): 17-27.

CHOQUETTE, L.P.E. et W.A. MOYNIHAN

1964 Control of Disease in Dogs in the Canadian North, Canadian Veterinary Journal, 5(10): 262-267.

CROTEAU, Jean-Jacques

2010 Rapport final de l'Honorable Jean-Jacques Croteau, juge retraité de la Cour supérieure relativement à son mandat d'examen des allégations d'abattage de chiens de traîneau inuits au Nunavik (1950-1970), Société Makivik.

DAMAS, David

2002 Arctic Migrants / Arctic Villagers. The Transformation of Inuit Settlement in the Central Arctic, Montréal et Kingston, McGill-Queen's University Press.

DIUBALDO, Richard J.

1985 The Government of Canada and the Inuit, 1900-1967, Ottawa, Department of Indian and Northern Affairs Canada, Corporate Policy, Research Branch.

DUHAIME, Gérard

1983 La sédentarisation au Nouveau-Québec inuit, Études/Inuit/Studies, 7(2): 2552.

FOUCAULT, Michel

1991 Faire vivre et laisser mourir. La naissance du racisme, Les Temps Modernes, 535: 37-61.

FREUCHEN, Peter

1935 The Eskimo Dog, in Mammals, Part II, Copenhague, Gyldendalske Boghandel, Nordisk Forlag, Report of the Fifth Thule Expedition 19211924, 2(4-5): 141-187.

GRC (GENDARMERIE ROYALE DU CANADA)

2006a The RCMP and the Inuit Sled Dogs (Nunavut and Northern Quebec: 19501970), Ottawa, Gendarmerie royale du Canada.

164/F. LÉVESQUE 
2006b Rapport final: Examen par la GRC des allégations relatives aux chiens de traîneau inuits, Ottawa, Gendarmerie royale du Canada.

JENNESS, Diamond

1964 Eskimo Administration: II. Canada, Montréal, Arctic Institute of North America.

LAUGRAND, Frédéric et Jarich OOSTEN

2002 Canicide and healing: The position of the dog in the Inuit cultures of the Canadian Arctic, Anthropos, 97(1): 89-105.

LESAGE, Jean

1955 Enter the European. V - Among the Eskimos (Part II), The Beaver, 285: 39.

LÉVESQUE, Francis

2008 Les Inuit, leurs chiens et l'administration nordique, de 1950 à aujourd'hui. Anthropologie d'une revendication inuit contemporaine, Thèse de doctorat, département d'anthropologie, Université Laval, Québec.

À paraitre An ordinance respecting dogs: How creating secure communities in the Northwest Territories made Inuit insecure, in Michelle Daveluy, Francis Lévesque et Jenanne Ferguson (dir.), Humanizing Security, Edmonton, CCI Press.

MALLON, Mick

s.d. The Teacher, the Dogs, and the Anthropologist, texte non publié en possession de l'auteur.

MacRURY, Ian Kenneth

1991 The Inuit Dog: Its Provenance, Environment and History, Mémoire de maîtrise, Études polaire, Darwin College, Scott Polar Institute, University of Cambridge.

QUMAQ, Taamusi

1992 L'avenir de l'Inuktitut, in Jacques Maurais (dir.) Les langues autochtones du Québec, Québec, Les publications du Québec, Dossier CLF 35: 343-360.

ROBERTSON, Gordon

1960 Administration for Development in Northern Canada: The Growth and Evolution of Government, Canadian Public Administration, 3(4): 354-362.

1961 The Future of the North, North, 8(2): 37-40. 
SALADIN D’ANGLURE, Bernard

1967 L'organisation sociale traditionnelle des Esquimaux de Kangirsujuaaq (Nouveau-Québec), Québec, Centre d'études nordiques, Université Laval.

1977 Iqallijuq ou les réminiscences d'une âme-nom inuit, Étude/Inuit/Studies, 1(1): 33-63.

\section{SOCIÉTÉ MAKIVIK}

2005a Mémoire au sujet de l'abattage de chiens inuits (qimmiits) au Nunavik, du milieu des années 1950 à la fin des années 1960, Présenté au ministre des Affaires indiennes et du Nord canadien du gouvernement du Canada et au ministre délégué aux Affaires autochtones du gouvernement du Québec, Société Makivik, janvier 2005 (En ligne à: http://pubs.aina.ucalgary. ca/makivik/CI232.pdf).

2005b Invitation aux médias, Communiqué de presse envoyé au média en janvier 2005 pour la première du film Echo of the Last Howl, Société Makivik, janvier 2005. 